\title{
Tensions entre ressources génétiques locales et ancrage territorial des produits. La race porcine corse dans un projet AOP
}

Tensions between local genetic resources and territorial anchorage of local products: Nustrale pig breed and Corsican pork butcheries PDO project case study

Adeline Lambert-Derkimba, Étienne Verrier et François Casabianca

\section{(2) OpenEdition}

\section{Édition électronique}

URL : http://journals.openedition.org/economierurale/2982

DOI : $10.4000 /$ economierurale.2982

ISSN : 2105-2581

Éditeur

Société Française d'Économie Rurale (SFER)

Édition imprimée

Date de publication : 21 mars 2011

Pagination : 39-49

ISSN : 0013-0559

Référence électronique

Adeline Lambert-Derkimba, Étienne Verrier et François Casabianca, «Tensions entre ressources génétiques locales et ancrage territorial des produits. La race porcine corse dans un projet AOP », Économie rurale [En ligne], 322 I mars-avril 2011, mis en ligne le 30 mars 2013, consulté le 19 avril 2019. URL : http://journals.openedition.org/economierurale/2982 ; DOI : 10.4000/ economierurale.2982 


\section{Tensions entre ressources génétiques locales et ancrage territorial des produits $L a$ race porcine corse dans un projet $A O P$}

Adeline LAMBERT-DERKIMBA - INRA, UR45 Laboratoire de recherches sur le développement de l'élevage, derkimba@supagro.inra.fr

Étienne VERRIER • INRA, AgroParisTech, UMR1313 Génétique animale et biologie intégrative, etienne.verrier@agroparistech.fr

François CASABIANCA • INRA, UR45 Laboratoire de recherches sur le développement de l'élevage, fca@corte.inra.fr

\section{Introduction}

L 'ancrage territorial des activités productives est souvent référé à la capacité des entreprises à construire des relations fortes avec leur environnement économique et technologique. Dans une telle perspective, l'économie des coûts de transaction (Riordan, Williamson, 1985) souligne l'importance de la notion d'actif. En tant qu'obstacle supposé à la délocalisation des activités, cette notion devient cruciale si l'on souhaite mieux appréhender les mécanismes d'ancrage territorial et leur mode de fonctionnement dans des Systèmes agroalimentaires localisés (Muchnik et al., 2008).

Nous proposons d'examiner le rôle des ressources territoriales dans le contexte de la protection des Indications géographiques (IG) vue comme facteur de localisation des activités. Parmi ces ressources, nous estimons qu'une place particulière doit être accordée aux ressources vivantes, présentes localement (ou non) dans leurs dimensions patrimoniales et dans leurs enjeux de gestion. En effet, si un actif montrant un haut degré de spécificité peut difficilement être redéployé, alors une race animale, même locale, composée d'animaux par définition mobiles et dont l'usage ne peut être réservé à une localisation particulière, semble pouvoir être aisément mobilisée en dehors de la zone considérée. Il est donc essentiel de bien élucider ce qui localise une race « locale » et d'en comprendre l'encastrement territorial à travers les systèmes de production qui, à la fois, la mobilisent et sont déterminés par elle.

De plus, la place conférée à une race locale peut conduire à une forme d'instrumentalisation où sa présence sur le territoire en fait une ressource captive, difficile à valoriser en dehors de l'IG qui en a fait une de ses justifications. D'où la formulation d'une dialectique entre race locale et IG qui la mobilise. Sous certaines conditions, le patrimoine génétique local peut concourir à l'ancrage territorial des systèmes agroalimentaires localisés (Bérard, Marchenay, 2006). Cependant, cet effet d'ancrage peut influencer en retour le devenir de la ressource génétique elle-même (LambertDerkimba et al., 2006). En d'autres termes, le fait qu'une ressource génétique locale soit activée par une filière sous IG, sans discuter du degré de spécificité d'un tel actif (Raynaud et al., 2005), montre des ambiguités dans ses conséquences sur le futur de la ressource. La ressource génétique, entendue ici comme une ressource territoriale vivante devient un enjeu d'appropriation (Linck, 2007) orienté par l'ancrage territorial des productions. Cet enjeu nourrit des négociations collectives complexes dont l'issue informe sur les dynamiques d'acteurs présentes dans le territoire et sur les éventuelles disparités de capital social (Shortall, 2008). Ainsi, notre hypothèse de recherche vise à associer étroitement l'activation de la ressource territoriale avec 
l'émergence de tensions entre les gestionnaires de cette ressource, d'une part, et les acteurs économiques qui la mobilisent dans leur projet, d'autre part.

Le cas étudié dans le présent article montre une réelle pertinence pour traiter de cette hypothèse. En effet, le porc en Corse a pendant longtemps été présent seulement à l'échelle familiale : quelques animaux de basse-cour destinés à l'autoconsommation. Aujourd'hui, la situation a évolué d'une économie d'autosubsistance vers une économie marchande (Bargain, 1989). La production fermière s'est développée, associant élevage extensif et transformation en charcuterie, et permettant aux éleveurs une rémunération accrue de leurs activités. La race porcine locale est reconnue par le ministère chargé de l'Agriculture et ses gestionnaires stabilisent actuellement leurs choix techniques. Dans ce contexte, un syndicat s'est créé pour déposer une demande officielle d'AOP Charcuteries de Corse. La race locale y est fortement mobilisée pour asseoir le lien du produit à son terroir de production. Il est ainsi nécessaire d'apprécier dans quelle mesure les gestionnaires de la ressource génétique, devenue un actif pour certains producteurs organisés, risquent de voir se réduire leur autonomie de décision.

Comme le montrent Cerdan et Fournier (2007), cette approche intéresse plus généralement la spécification des ressources territoriales dans sa capacité à produire le Système agroalimentaire localisé (SYAL) lui-même. Ce phénomène est donc considéré comme au cœur des rapports entre ressources et projets territoriaux. Dans une approche globale des Systèmes agroalimentaires localisés, le territoire est vu comme "un acteur stratégique potentiel capable de combiner et activer ces ressources, un facteur d'assemblage d'activités pour incrémenter leur compétitivité » (Muchnik et al., 2008). Notre approche vise donc à mieux informer des tensions qui se font jour dans de telles constructions, alors même qu'elles sont le plus souvent minorées par les observateurs au profit de leurs conséquences positives en termes de coordination et de confiance.

Après une présentation du contexte régional et de sa dynamique historique, nous nous attachons à rendre compte de la manière dont la race est convoquée dans le projet d'AOP, ce qui nous permet de contextualiser notre hypothèse et de formuler les principales tensions identifiées. Cela nous conduit à mettre en place un dispositif de recueil de données originales orientées par ces tensions. Enfin, nous discutons, à partir de la mobilisation des races locales dans de tels projets, des enseignements généralisables pour l'approche de l'activation des ressources territoriales.

\section{Le contexte régional et sa dynamique historique}

On trouve aujourd'hui une grande diversité de produits de charcuterie sous la dénomination «Corse ». Actuellement, les éleveurs de porcs sont majoritairement à la fois producteurs et transformateurs (De Sainte Marie et al., 1998). Les industriels, quant à eux, s'approvisionnent en grande partie sur le marché européen du porc maigre avec un coût moindre que le prix de revient local et une plus grande disponibilité. Différentes initiatives ont tenté, sans succès jusqu'à maintenant, de valoriser un produit local issu de systèmes d'élevage traditionnels en le distinguant des autres productions.

\section{Valorisation de la race et des produits}

En 1990, une Société d'initiative et de coopération agricole, la SICA Testa Nera, se crée dans l'optique d'identifier une charcuterie corse de très haut de gamme (De Sainte-Marie, Casabianca, 1998). Elle met alors en place un cahier des charges (le premier du genre en Corse) que chaque éleveur adhérent doit observer (phénotype des animaux, conduite d'élevage, procédés de transformation et d'affinage). Malgré la 


\section{Figure 1. Évolution des démarches de valorisation de la race et des produits}

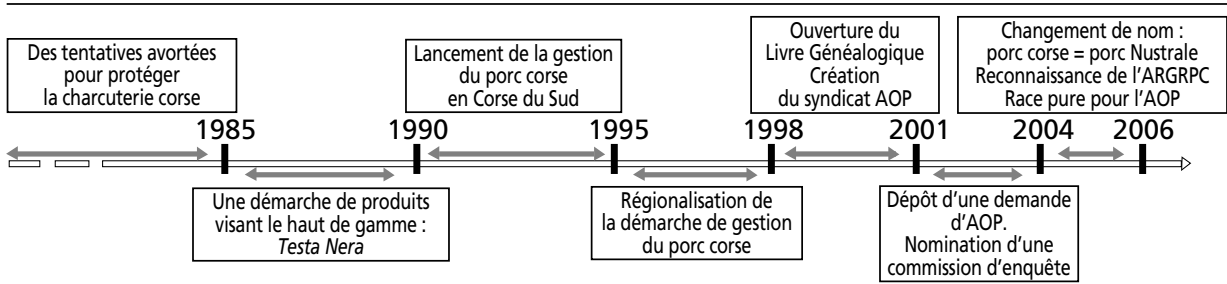

grande valeur ajoutée de ces produits de luxe, cette démarche échouera à la suite de vols successifs. Ainsi, l'idée d'une protection de la charcuterie perdure, mais sans faisabilité suffisante pour engager de projet collectif.

Toutefois, la démarche de Testa Nera a amorcé une dynamique autour de l'enjeu patrimonial que représente la race locale de porc. En 1994, à partir des producteurs de la SICA, une association départementale d'éleveurs se crée en Corse du Sud (Saby, 1995), puis devient en 1998 l'Association régionale de gestion de la race porcine corse (ARGRPC). Son objectif est triple : définir les orientations de la race porcine corse, coordonner les actions de sélection la concernant et faire reconnaître la race par le ministère chargé de l'Agriculture. Son travail commence par une définition du standard de la race (Casabianca et al., 2000) qui se fera aux dires d'acteurs, avec l'appui de chercheurs et de techniciens et permet l'ouverture du Livre généalogique (LG) annexe à partir de fondateurs identifiés (inscription au Livre généalogique des races locales de porcs - LIGERAL).

\section{La construction du projet AOP}

\section{Sur les traces de la réussite raciale}

À la suite de ces démarches, la possibilité de promouvoir une AOP en charcuterie fait son chemin dans les esprits de certains acteurs locaux. L'ouverture du LG principal en 2001 (inscription d'animaux comprenant trois générations de reproducteurs conformes au standard) induit quelques mois plus tard la création du syndicat de défense et de promotion de la charcuterie corse (figure 1). La démarche de gestion de la race locale crédibilise progressivement la perspective de certification des produits de charcuterie.

La place que peut prendre la race locale dans la construction de la future AOP est objet de réflexion collective. De nombreux débats sont ouverts au sein du syndicat afin de définir le règlement technique. Après avoir choisi les produits qu'il fallait protéger (seules les pièces entières dans un premier temps : prisuttu, lonzu, coppa ou jambon sec, échine et longe salée et séchées), deux éléments paraissent fondamentaux dans les débats : les types génétiques à considérer et l'alimentation des animaux en finition.

\section{La race prise dans la construction des conditions de production de I'AOP}

\section{Les points d'appui d'une légitimation de la demande d'AOP}

Dans le système traditionnel les animaux trouvaient de quoi assurer leur finition dans les châtaigneraies et les chênaies (Agostini, 1989). Aujourd'hui, les ressources naturelles sont plus limitées et les animaux ne peuvent plus dans leur totalité se nourrir de châtaignes et de glands en finition. Ils sont donc généralement nourris en partie, voire exclusivement, avec de l'orge. Le choix du collectif a été de ne pas exclure les éleveurs ayant recours à l'orge, sans que cela remette en question le lien au terroir. La proposition était donc d'autoriser une finition selon trois modalités possibles : finition strictement à la châtaigne ou aux glands, finition à la châtaigne ou aux glands avec complémentation en orge si nécessaire et finition à l'orge. 
En parallèle, la question de la race locale, élément concret de motivation pour entrer dans la démarche AOP, a été l'autre point important des débats sur la construction du cahier des charges. Faut-il favoriser les animaux de race pure corse, ou bien est-il possible de croiser des femelles de race corse avec des mâles de races spécialisées dans la production de viande maigre, comme le Large White ou le Duroc ? Le croisement en élevage porcin étant une pratique attestée depuis de nombreuses années en Corse, la seconde solution permet aux éleveurs de se conformer aux usages locaux.

\section{L'instruction par l'Institut national des appellations d'origine perturbe les compromis locaux}

Le projet d'AOP déposé en 2004 à l'Institut national des appellations d'origine (INAO) proposait donc deux types génétiques possibles : soit un porc Corse ${ }^{1}$ de race pure, soit un porc issu du croisement de première génération entre truie Corse et verrat d'une autre race. Il proposait également deux modes de finition : soit orge seul ou en mélange, soit châtaigne et/ou gland exclusifs. À ces conditions de production, s'ajoutaient des valeurs seuils de poids de carcasse permettant de définir le format des animaux à un âge minimum donné. Ces choix étaient analogues à ceux retenus par les producteurs espagnols pour protéger les produits issus de leur race locale ibérique (Lopez-Bote, 1998). Mais, là où le collectif visait une diversité au sein de l'AOP, la commission d'enquête de l'INAO a invoqué la nécessité de conditions de production identiques pour tous les producteurs et d'un produit relativement homogène, facilement contrôlable au sein de la filière, et aisément identifiable par les consommateurs. Elle a donc refusé, lors de sa première venue en 2006, des

1. Afin de faciliter la lecture, nous conserverons l'appellation porc Corse tout au long de cet article, appliquée également aux autres porcs régionaux. différenciations au sein même de l'AOP et les producteurs ont dû choisir entre fixer le type génétique ou fixer le type d'alimentation en finition. À la suite de nombreux débats, le choix s'est porté sur la fixation de la race utilisée, avec plus de flexibilité sur l'alimentation. De plus, et à la demande de l'INAO, en vertu du règlement européen de 1992 qui vise à éviter toute confusion entre les noms de la race et du produit à protéger, le syndicat requiert de la part de l'ARGRPC un changement du nom de la race : la race corse devient donc race Nustrale en 2006, succédant à une décision prise par les gestionnaires de la ressource génétique, tous intéressés également à l'AOP.

Le choix d'inclure l'orge répond à une stratégie adaptative. La question de la châtaigneraie en Corse est un sujet complexe impliquant un grand nombre d'acteurs (De Casabianca, Santucci, 1989), avec des enjeux fonciers délicats. La fluctuation des rendements en châtaignes et en glands selon les années, ne permet pas non plus d'en faire une ressource stable, et les risques de déclassement des carcasses pour cause de pénurie de châtaignes seraient trop importants. On peut ajouter que la gestion d'une race peut sembler plus aisée que celle de la châtaigneraie et que l'utilisation exclusive de la race corse est certainement plus porteuse en termes d'image.

\section{Mise en contexte de notre hypothèse}

Le projet d'AOP est désormais fondé sur l'utilisation exclusive d'animaux de race pure, sans ajustement des valeurs seuils de poids de carcasse qui permettait de valider des animaux issus de croisement. Notre hypothèse de recherche nous conduit à identifier deux groupes de tensions engendrées par les dynamiques à l'œuvre.

- Comment va évoluer le caractère de croissance lente propre à la race locale dans une population mobilisée en grande partie par une AOP autorisant l'usage de l'orge dans l'alimentation, ce qui permet l'obten- 
tion de poids de carcasse élevés ? Plus généralement, un tel couplage entre race locale et projet d'AOP questionne-t-il la définition même de la race et des caractères à sélectionner ?

- Comment va évoluer le marché des reproducteurs d'animaux de race pure face à la forte demande induite par l'AOP ? Où plus généralement, quelle interprétation donner au terme « race » dès lors que son usage devient obligatoire?

\section{Un dispositif de recueil de données}

Pour traiter ces questions, nous avons conduit 15 entretiens semi-directifs (Ghiglione, Matalon, 1970) auprès d'éleveurs gestionnaires de la race et/ou engagés dans le projet d'AOP, avec retranscription des discours, entre 2005 et 2007. Sur la même période, nous avons également réalisé une observation participante sur une quarantaine de réunions d'instances professionnelles : comités techniques et conseils d'administration de l'ARGRPC et du syndicat AOP. Il nous a ainsi été possible de noter les arguments techniques avancés par les différents acteurs engagés et de repérer les controverses sur les choix réalisés aussi bien pour la gestion de la race que pour l'élaboration du projet AOP.

\section{Croissance lente Une caractéristique raciale face aux conditions de I'AOP}

\section{Le potentiel génétique en question}

La bibliographie fournit des éléments sur des races dites rustiques, dont la croissance lente est une des caractéristiques, comme le porc Gascon ou le porc Basque (Labroue, 1998). Une comparaison avec six autres races ou lignées spécialisées (Gascon, Limousin, Basque, Ibérique, Meishan, Large White Landrace) permet d'établir que le porc Corse a une croissance lente : alimenté ad libitum, il montre la valeur la plus faible pour le gain de poids et la valeur la plus élevée pour l'âge à $100 \mathrm{~kg}$, avec toutefois des profils de croissance d'allure similaire à ceux des autres races; et son indice de consommation (mesuré par la quantité d'aliment complet nécessaire au croît d'un kilo de poids vif) est parmi les plus élevés avec une valeur de 6.7 (Secondi, 1996).

Pour certains gestionnaires de la race, la croissance lente est une caractéristique essentielle, un élément identitaire, du porc de race locale. Des mesures de croissance de jeunes verrats dans les années 1990 (Casabianca et al., 2000) ont conduit à éliminer des sujets à croissance « trop » rapide (plus de $80 \mathrm{~kg}$ de poids vif à 6 mois d'âge), considérés alors comme croisés. Mais seule une minorité d'usagers de la race accorde à cette caractéristique une origine génétique : à partir d'observations d'animaux de même portée élevés dans des élevages différents, beaucoup considèrent qu'elle résulte des seules pratiques d'élevage. On peut donc s'interroger sur la place qui peut être faite à la vitesse de croissance dans les objectifs de sélection.

\section{Confrontation aux conditions de production de la future AOP}

Les règles proposées dans le dossier d'AOP ont été conçues au moment où le croisement était autorisé, ce qui donne une gamme de valeurs seuils de poids de carcasse assez large : âge de 12 à 36 mois ; poids de carcasse de 80 à $140 \mathrm{~kg}$. Ces valeurs sont à comparer avec le champ de variation actuel du poids à l'abattage des porcs charcutiers de race corse, les éleveurs interrogés estimant que ces poids vont de 75 à $130 \mathrm{~kg}$. Les éleveurs s'inscrivant dans une démarche raciale déclarent obtenir des poids de carcasse plus faibles que les autres éleveurs disposant souvent d'animaux croisés. Les valeurs du projet AOP autorisent ainsi la conformité d'animaux du format des croisés.

Cette situation ne risque-t-elle pas d'entraîner un changement d'orientation des systèmes de production, traditionnellement fondés sur la production au moindre coût 
valorisant les espaces naturels ? Et que devient le caractère de croissance lente de la race locale?

\section{Quelles valeurs seuils de la croissance lente ?}

Les performances de production dépendent entre autres de la quantité et de la qualité de l'aliment, et de la capacité de l'animal à valoriser ce dernier. Accroître le potentiel de croissance des animaux répond à la nécessité de compenser les frais engagés pour nourrir les animaux pendant l'estive et éventuellement pendant la finition, et n'entraîne pas forcément une exclusion de l'AOP puisque les seuils de poids de carcasse le permettent. Notons qu'avec le porc Corse de race pure, qui a un indice de consommation très élevé ( $c f$. plus haut), l'alimentation à base d'orge sera coûteuse. Les éleveurs dépourvus de châtaigneraies ou de chênaies pour alimenter leur troupeau en période de finition peuvent alors s'inquiéter des surcoûts de production liés à cette race locale incapable de bien valoriser l'alimentation apportée.

Si la gestion génétique de la race peut être fortement orientée par les intérêts s'exprimant dans l'AOP, on peut penser qu'alors, la capacité à bien valoriser l'alimentation à base d'orge pourrait être une des caractéristiques à améliorer dans la race pure. À cela, s'ajoute la possibilité de formats de carcasses supérieurs à ce que la race pure exprime généralement. Un des leviers qu'il est possible d'actionner est donc la pression de sélection, permettant d'améliorer le potentiel de croissance des animaux et de valoriser l'alimentation qui leur est distribuée. La gamme de poids autorisée par le cahier des charges laisse une large marge de manœuvre pour travailler sur ce caractère.

Les éleveurs-sélectionneurs ne vont-ils pas choisir comme objectif pour la race Corse de rejoindre les performances actuelles des porcs croisés en matière d'efficacité alimentaire et de vitesse de croissance ? Cela pourrait remettre en cause la définition de la race locale, avec une ques- tion encore peu facile à instruire : quel est le seuil maximum de la croissance dite « lente »? La définition des objectifs de sélection de la race est cadrée par l'activation de la ressource au sein de l'AOP (à travers le cahier des charges). Si cette définition n'est pas encore construite et fait aujourd'hui l'objet d'un évitement permanent au sein des instances de gestion de la race, elle va pourtant devoir être traitée rapidement et représente un réel enjeu pour le devenir de la ressource et du projet AOP.

\section{Projet d'AOP et dynamique du marché des reproducteurs}

\section{Race pure: des animaux apparentés exclus du système}

Lors de la mise en place du Livre généalogique, les éleveurs ont choisi des animaux fondateurs parmi la population conforme au standard. Ces animaux constituaient pour les éleveurs un échantillon représentatif des différentes lignées et familles existantes, et seuls ces quelques individus ont été retenus ( 5 femelles maximum par exploitation). En effet, les agents en charge de ce travail ont décidé de favoriser une diversité d'origine génétique sans surcharger le travail de saisie et de gestion du fichier. Pour les éleveurs ayant fourni les animaux fondateurs pour le Livre, des animaux ont donc été exclus malgré leur conformité au standard.

Or, le rôle des truies dans l'exploitation du territoire pastoral est central par leur connaissance des aires d'alimentation, d'abreuvement et de couchage. Rendues toutes complémentaires entre elles (déplacement par groupe avec truies meneuses), ces truies transmettent ensuite ce savoir à leur descendance par apprentissage et assurent un usage de tout le territoire. Pour les éleveurs, il est impossible de se séparer d'une partie de ces truies, surtout si les «meneuses » ne sont pas celles inscrites au LIGERAL. Les éleveurs sont ainsi mécontents de n'avoir qu'une partie du troupeau reconnue de race Corse et potentiellement 
apte à produire des porcs pour l'AOP. Le dilemme qui se pose est clair : que faire des animaux non inscrits au LG mais présentant les caractéristiques d'un porc de race locale?

\section{L'exclusivité de la race pure en question}

Le syndicat AOP explore alors une solution pour mettre fin à la difficulté soulevée par l'exclusion d'un grand nombre d'animaux. Il propose d'associer au cahier des charges, des animaux inscrits à un registre identifiant un type racial fondé sur le phénotype (tableau 1). L'objectif est de permettre à un maximum d'animaux d'être éligibles pour l'AOP et d'assurer un volume de production suffisant. C'est l'ARGRPC qui est pressentie pour prendre en charge ce registre. L'autorisation d'animaux de type racial (inscrits au registre) interroge sur la mise en concurrence qui est alors créée autour des animaux de race pure. L'exclusivité de la race pure pour la production AOP créait de la rareté et avait donc une influence positive sur la valorisation marchande de la ressource. Mais l'autorisation du type racial fait disparaître cette valeur et met la race pure en concurrence avec les animaux de type racial. Le petit noyau d'animaux de race pure ne risque-til pas d'être mis en péril par cette proposition du syndicat?

\section{Race pure ou type racial ?}

L'arbitrage entre race pure et type racial prend en compte les processus et les coûts de production des différents types d'animaux : (i) la gestion génétique coûte cher, selon la qualité de l'animal, en particulier pour les mâles, (ii) la prise en compte de l'origine génétique des animaux dans le système d'exploitation induit des contraintes dans les pratiques d'élevage et de gestion des accouplements et (iii) la surveillance pour contrôler les filiations maternelles est nécessaire. La production d'un animal de type racial n'implique pas ces coûts spécifiques à la production de race pure. En outre, la question de l'accessibilité se pose : l'achat de reproducteurs de race pure oblige l'accès à des réseaux nouveaux (coûts de transaction). La possibilité de profiter des outils collectifs, dont l'Insémination artificielle (IA), dépend également de l'accès à ces réseaux. L'information sur les reproducteurs eux-mêmes devient plus coûteuse, en comparaison des animaux de type racial dont l'approvisionnement est assuré par des réseaux anciens d'échanges de reproducteurs.

La comparaison entre race pure et type racial en termes de coûts et d'accessibilité donne largement l'avantage au type racial. Une question majeure se pose alors : quelle motivation pour les éleveurs à investir dans des animaux de race pure si des animaux de type racial donnent accès à l'AOP ? L'ARGRPC a logiquement refusé la responsabilité de constitution de ce registre, ce qui s'est traduit par une tension explicite avec le syndicat. Dans une forme de réponse à ces sollicitations, l'ARGRPC a élaboré des règles d'enregistrement et d'usage destinées à réguler l'utilisation des reproducteurs en s'assurant l'exclusivité de leur fourniture. La tension ainsi nouée montre clairement que l'activation de la race locale révèle des enjeux liés à l'appropriation d'un bien commun jusqu'alors vu comme libre d'accès et sans restriction d'usage.

Tableau 1. Différences majeures entre race pure et type racial

\begin{tabular}{l|l|l}
\hline Ressource & Race pure & Type racial \\
\hline Identification des animaux & Livre généalogique : généalogie et standard & Registre : standard \\
\hline Gestion génétique & $\begin{array}{l}\text { Gestion des caractères morphologiques } \\
\text { et de production, gestion de la variabilité } \\
\text { génétique }\end{array}$ & $\begin{array}{l}\text { Gestion des caractères } \\
\text { morphologiques }\end{array}$ \\
\hline
\end{tabular}




\section{Discussion}

L'analyse du cas de la filière porcine en Corse montre que le couplage race locale / IG engendre effectivement des enjeux majeurs pour le futur de la ressource génétique. La mise en tension de la caractéristique de croissance lente par les critères du projet AOP souligne son impact qualitatif potentiel. La mise en concurrence de la race pure par le type racial comme alternative de production souligne son impact quantitatif possible. L'influence observée peut également interroger sur l'appropriation de la ressource génétique par le collectif AOP et sa légitimité pour ce faire. L'acteur organisé, soutenu par les institutions territoriales, que constitue le syndicat AOP peut-il prétendre définir à lui seul la ressource génétique de demain ? La race locale se doit alors d'être approchée en tant que bien commun soumis à des tentatives d'appropriation au nom d'un intérêt général (Lascoumes, Le Bourhis, 1998) bien compris.

Notre étude de cas met ainsi en lumière ce qui se joue dans le fait de rendre obligatoire l'usage d'un actif comme une ressource génétique locale, construite à travers un dispositif de gestion localisé et rendre ainsi cet actif mobilisable pour un projet territorial. Le statut des animaux de la race locale en est profondément changé. Le rôle des sélectionneurs, leur place dans le système régional, les critères auxquels on va soumettre leur action collective pour l'évaluer à travers les attentes du projet territorial, tout cela est largement bouleversé. Ainsi, entre ressource et actif, comme le montrent Benko et Pecqueur (2001), ce sont des ensembles de positions, de valeurs et de capacités d'action qui sont redistribués au sein des territoires.

De nombreuses tensions apparaissent dans un tel mouvement.
Notre étude de cas identifie quatre questions :

- Le nom ;

- les critères pour orienter la race ;

- les droits de propriété ;

- les légitimités et le pouvoir de marché.

- La question du nom. Les conflits potentiels de dénomination incluant un localisant ont reçu un traitement déjà vu dans des situations analogues : c'est la race qui abandonne son nom au profit du produit alimentaire qui va apparaître sur le marché, comme ce fut le cas des races bovines Maine-Anjou et Camargue devenues Rouge des Prés et Raço di Biou, respectivement. Une telle décision suppose un niveau de compréhension mutuelle qui n'est pas forcément toujours atteint.

- La question des critères pour orienter la race. Concevoir un ensemble d'indicateurs permettant de gérer collectivement la race est effectivement modifié par la mobilisation de cette race dans un projet territorial. Une telle modification peut révéler des contradictions (telles que celles qui apparaissent sur la question de la croissance lente).

- La question des droits de propriété et des droits d'usage. Les notions de «sélectionneurs » et d'utilisateurs de la race forment la trame d'une redistribution des responsabilités, des enjeux de valorisation et d'équilibre économique des exploitations, mais aussi des effets de rareté ou de situation d'otage souvent décrits dans la littérature (Boissin, 1999).

- La question des légitimités et du pouvoir de marché, pour disposer des moyens nécessaires. Cette question est liée au fait que le marché des reproducteurs n'a pas la même portée que le marché des aliments, y compris, en termes de possibilité de mobiliser des fonds pour s'organiser et pour faire valoir ses intérêts. 
Cette dernière question du capital social (Shortall, 2008) souligne que le projet d'AOP draine plus largement dans la société locale alors que le travail des éleveurs de la race demeure dans l'ombre et sans publicisation automatique. Elle nous semble assez généralisable. En effet, la race ne concerne pas directement l'assiette des consommateurs. Toutefois, dans notre cas, les coordinations locales demeurent fortes et le fait que de nombreux acteurs appartiennent de fait aux deux projets (et jouent ainsi un rôle de médiateurs) a permis de limiter les zones de conflits et de gérer les tensions qui sont apparues. Comme le souligne Courlet (2007), la densité institutionnelle peut alors être identifiée comme un facteur de régulation des conflits.

De telles explorations nous semblent apporter des éléments généralisables à de nombreuses situations où la ressource territoriale (Gumuchian, Pecqueur, 2007) une fois engagée dans des projets, pose la question de l'appropriation d'un bien commun (Linck, 2007).

\section{Conclusion}

Au terme de notre analyse, nous confirmons que l'activation d'une ressource comme facteur d'ancrage territorial est un processus complexe générateur de tensions au sein des territoires. Elle transforme la ressource en actif et identifie par là même de nouveaux enjeux pour le développement territorial. Cette activation représente à la fois des opportunités qui s'ouvrent et des dangers potentiels pour le futur de la ressource. Elle attise les enjeux d'appropriation de la ressource territoriale tout en lui contribuant une valeur nouvelle.

Notre exploration débouche sur le besoin d'identifier des espaces de médiation pour résoudre les tensions entre les gestionnaires de la ressource et les porteurs du projet.
De tels espaces de médiation rendent possible l'élaboration des arbitrages nécessaires pour surmonter les contradictions d'intérêt de chacun. En particulier, le fait que certains acteurs puissent faire partie des deux ensembles leur permet de jouer un rôle d'explicitation des raisons profondes qui sous-tendent les tensions identifiées (Lambert-Derkimba, 2007). Cette opportunité n'est pas toujours présente et le besoin peut alors se faire sentir de créer des instances où les représentants des projets territoriaux sont légitimés pour faire valoir leur point de vue sur la gestion de la ressource.

Enfin, l'activation est à interpréter comme l'inscription de la ressource dans du court terme, alors que la patrimonialisation nourrie par les gestionnaires de la race locale revient à l'inscrire dans des processus inter-générationnels. Ce paradoxe des temporalités est à affronter car une telle situation d'activation dans laquelle une ressource se transforme en actif est assez générale dans les SYAL et souvent donnée comme forcément positive pour l'ensemble des acteurs. Une attention particulière devra donc être portée à cette transformation dans la mesure où elle ne semble pas vraiment anodine ni s'inscrire spontanément dans des relations synergiques. Elle se révèle même capable d'affecter le futur de la ressource en contraignant ses gestionnaires à abandonner tout ou une partie de leur capacité d'orientation au profit des seuls acteurs des filières.

Le premier auteur a bénéficié d'une bourse de recherche financée par la Collectivité territoriale de Corse et par les départements SAD (Sciences pour l'action et le développement) et GA (Génétique animale) de I'INRA (Institut national pour la recherche agronomique). Les auteurs remercient Emmanuel Raynaud pour sa relecture et ses commentaires, en particulier sur les cadres théoriques. Nous remercions aussi les deux relecteurs et les éditeurs de ce numéro spécial. (NDA) 


\section{RÉFÉRENCES BIBLIOGRAPHIQUES}

Agostini P. (1989). La maîtrise de l'élevage porcin extensif dans le cadre du développement intérieur de la Corse. Corte, Mémoire Universita di Corsica. Bargain V. (1989). Étude des systèmes d'élevage porcin de la région Corse. Montpellier, Mémoire ENSA.

Benko G., Pecqueur B. (2001). Les ressources de territoires et les territoires de ressources. Finisterra, XXXVI, n ${ }^{\circ} 71$, p. 7-19.

Bérard L., Marchenay P. (2006). Local products and geographical indications: taking account of local knowledge and biodiversity. International Social Science Journal. Cultural Diversity and Biodiversity, $\mathrm{n}^{\circ} 187$, p. 109-116.

Boissin O. (1999). La construction des actifs spécifiques. Une analyse critique de la théorie des coûts de transaction. Revue d'Économie Industrielle. Vol. 90, p. 7-24.

Casabianca F., Poggioli A., Rossi J.-D., Maestrini O. (2000). L'amorce d'une gestion collective de la race porcine Corse. Construire un standard et élaborer les contrôles des reproducteurs. In Options méditerranéennes : «Tradition and innovation in Mediterranean pig production », vol. 41, p. 23-34.

Cerdan Cl., Fournier St. (2007). Le système agroalimentaire localisé comme produit de l'activation des ressources territoriales. Enjeux et contraintes du développement local des productions agroalimentaires artisanales. In Gumuchian $\mathrm{H}$, Pecqueur B., (dir.), «La ressource territoriale », Economica, Anthropos, p. 104123.

Courlet C. (2007). Du développement économique situé. In Gumuchian H., Pecqueur B. (dir.), «La ressource territoriale », Economica, Anthropos, p. 3244.

De Casabianca F., Santucci P. (1989). Une expérience de recherche-développement en zone marginale : la Castagniccia. Greghje e rughjoni, $\mathrm{n}^{\circ}$ 16, p. 151-165. De Sainte-Marie C., Casabianca F. (1998). Entre logique individuelle et intégration : la « fruitière » comme modèle d'organisation pour des producteurs fermiers de charcuterie en Corse. Études et Recherches sur les Systèmes Agraires et le Développement, $\mathrm{n}^{\circ} 31$, p. 297-315.

De Sainte-Marie C., Casabianca F., Poggi M. (1998). Les activités d'élevage porcin en Corse. Des difficultés de la transition entre économie souterraine et économie formelle. In Italiane E. S. (éd.) «Corsica. Isola problema tra Europa e Mediterrane », p 263-285.

Ghiglione R., Matalon B. (1970). Les enquêtes sociologiques, théories et pratiques. Paris, Armand Colin.

Gumuchian H., Pecqueur B. (2007). La ressource territoriale. Economica, Anthropos, 252 p.

Labroue F. (1998). Étude comparative de quatre races locales pour les performances de croissance, carcasse et qualité de la viande. Le Rheu, ITP.

Lambert-Derkimba A. (2007). L'inscription des races locales dans les conditions de production des produits animaux sous AOC : enjeux et conséquences pour la gestion collective des races mobilisées. Paris, Thèse de doctorat, AgroParisTech, $265 \mathrm{p}$.

Lambert-Derkimba A., Casabianca F., Verrier E. (2006). L'inscription du type génétique dans les règlements techniques des produits animaux sous AOC : conséquences pour les races animales. INRA Productions Animales, ${ }^{\circ}$ 19, p. 357-370.

Lascoumes P., Le Bourhis J.-P. (1998). Le bien commun comme construit territorial. Identités d'action et procédures. Politix, vol. 11, n 42, p. 37-66.

Linck T. (2007). Le dilemme de la gestion patrimoniale. L'exclusion, condition et 
obstacle à l'appropriation collective. Économie Appliquée, LX (3), p. 177-198.

Lopez-Bote Cl. (1998). Sustained utilization of the Iberian pig breed. Meat science, vol. 49, Suppl. 1, S17-S27.

Muchnik J., Sanz Canada J., Torres Salcido G. (2008). Systèmes agroalimentaires localisés : état des recherches et perspectives. Cahiers Agricultures, vol. $17, \mathrm{n}^{\circ} 6$, p. 513-519.

Raynaud E., Sauvée L., Valceschini E. (2005). Alignment between Quality Enforcement Devices and Governance Structures in the Agro-food Vertical Chains. Journal of Management and Governance, $\mathrm{n}^{\circ}$ 9, p. 47-77.

Riordan M., Williamson O. (1985). Asset specificity and economic organization.
International Journal of Industrial Organization, $\mathrm{n}^{\circ} 3$, p. 365-378.

Saby C. (1995). Contribution à la conception et à la mise en place d'un schéma de sélection d'une race locale: le cas $d u$ porc Corse. Paris, Mémoire de fin d'études, Institut National Agronomique Paris - Grignon.

Secondi F. (1996). Croissance, développement tissulaire et caractéristiques de la carcasse du porc Corse. Journées de la Recherche Porcine, $n^{\circ}$ 28, p. 109-114. Shortall S. (2008). Are rural development programmes socially inclusive? Social inclusion, civic engagement, participation, and social capital: Exploring the differences. Journal of Rural Studies, $\mathrm{n}^{\circ} 24$, p. $450-457$. 